\title{
SIMULACIÓN CON EASY JAVA SIMULATIONS Y RENDIMIENTO ACADÉMICO DE LOS ESTUDIANTES DE ANÁLISIS MATEMÁTICO
}

\author{
SIMULATION WITH EASY JAVA SIMULATIONS AND ACADEMIC PERFORMANCE OF MATHEMATICAL ANALYSIS \\ STUDENTS
}

\author{
Jaime Rodrigo Guilcapi Mosquera ${ }_{2}^{1}$ \\ Rafael Santiago Albuja Echeverria ${ }^{2}$ \\ Franklin Marcelo Coronel Maji ${ }^{3}$ \\ Freddy Geovanny Benalcázar Palacios ${ }^{4}$
}

\section{Resumen}

Se implementa el Easy Java Simulations (EJS) como instrumento de apoyo en el proceso de enseñanza aprendizaje de los estudiantes de análisis matemático. Inicialmente se identifican las variables involucradas en el proceso de enseñanza - aprendizaje que desarrollan los estudiantes de la materia de análisis matemático, así como las dificultades que estos tienen, para con ello estructurar una guía de uso del EJS. La propuesta desarrollada permite obtener una aproximación teórica - práctica, identificando aquellos elementos que pueden ser de utilidad y aporte tanto para el docente como para el estudiante en el proceso de enseñanza aprendizaje de la matemática a través del EJS. Para culminar se comprueba que existen diferencias significativas entre un grupo de control y un grupo de intervención, al cual se implementó EJS, en el proceso de enseñanza aprendizaje de análisis matemático. Se concluye un efecto positivo en el rendimiento académico.

\section{Palabras clave}

Análisis matemático, inter-aprendizaje, software Easy Java Simulations.

\section{Abstract}

Efforts are being made to implement easy java simulations as a support instrument in the teaching-learning process of the students who take the subject of mathematical analysis III. The variables involved in the teaching and learning processes developed by the students to approve the subject of mathematical analysis are detailed, as well as the difficulties they have, in order to structure a guide for the use of easy java simulations. The developed proposal allows obtaining a theoretical - practical approach, identifying those elements that can be useful and contribution both for the teacher and for the student in the teaching-learning process of mathematics through easy java simulations. To conclude, we verified that there are significant differences between a control group and an intervention group, that is, the implementation of the easy java simulations software in the teaching-learning process of mathematical analysis if it positively influences academic performance.

\section{Keywords}

Mathematical Analysis, inter-learning, Software Easy Java Simulations.

\footnotetext{
${ }^{1}$ Universidad Técnica de Ambato. (Ecuador). Correo electrónico: jr.guilcapi@uta.edu.ec.

${ }^{2}$ Escuela Superior Politécnica de Chimborazo (Ecuador). Correo electrónico: ralbuja@espoch.edu.ec.

${ }^{3}$ Escuela Superior Politécnica de Chimborazo (Ecuador). Correo electrónico: fmcoronel9@outlook.com.

${ }^{4}$ Universidad Técnica de Ambato (Ecuador). Correo electrónico: fg.benalcazar@uta.edu.ec.
} 


\section{Introducción}

Las Tecnologías de la Informática y las Comunicaciones (TIC) y los materiales educativos computarizados (MEC's) son una realidad en el mundo de hoy. Rodean al ciudadano común por donde quiera que se encuentre, ya sea en su empresa o en un banco, en un hospital o en un centro deportivo o cultural. Más aún, se puede decir que invaden la intimidad del hogar a través de la telefonía, la televisión, el internet, o de una ingeniosa herramienta como lo es la computadora personal (PC). Especialmente, la utilización de computadores personales pueden contribuir al desarrollo cognitivo, al permitir el acceso a una cantidad considerable de información, así como a una valiosa interactividad del estudiante con el equipo, a través de softwares de aplicación general o mediante asistentes matemáticos de distinto tipo. Los docentes no pueden ignorar este avance tecnológico. Deben formar parte de este moderno esquema educativo, y lograr que sus estudiantes asuman los retos que impone la ciencia y la tecnología. Grandes cambios científicos necesitan individuos con un alto nivel cognitivo, creativo, y con capacidades mentales desarrolladas.

Sin embargo, las instituciones de educación superior ecuatorianas, y especialmente la Escuela Superior Politécnica de Chimborazo (ESPOCH), han sido ajenas a las metodologías de enseñanza-aprendizaje basadas en las nuevas tecnologías de la información, así como en el uso de materiales educativos computacionales, a pesar de que han realizado procesos de mejoramiento de su oferta académica y distintos rediseños curriculares. En el caso específico de la asignatura de análisis matemático III, que se imparte en distintas carreras de la ESPOCH, los aspectos en los que mayor dificultad presentan los estudiantes corresponden a la formulación y resolución de modelos matemáticos aplicados a fenómenos geométricos, físicos y otros, los cuales no son perceptibles fácilmente, dificultad que se agudiza por la no utilización de métodos interactivos y modernos, como lo sería la utilización de un software adecuado para facilitar el estudio de dicha temática.

En este marco, el presente estudio pretende describir y analizar la implementación del software Easy Java Simulations (de ahora en adelante EJS) como instrumento de apoyo en el proceso de enseñanza aprendizaje de los estudiantes de análisis matemático. Se pretende presentar una aproximación teórica - práctica, identificando aquellos elementos que pueden ser de utilidad para el docente como para el estudiante. En términos concretos, se pretende establecer una relación positiva y significativa entre el uso del EJS para el aprendizaje de los contenidos de la asignatura de análisis matemático III y el rendimiento académico de los estudiantes.

\section{Metodología}

El proceso investigativo consistió, en primer lugar, en determinar el conocimiento de la guía y software a utilizarse por parte de los docentes participantes en la investigación, o de cualquier otra herramienta metodológica fuera de las tradicionales, que a los mismos les facilite y ayude en el proceso enseñanza-aprendizaje de las matemáticas, pues su criterio sería fundamental para determinar si desde su punto de vista este tipo de herramientas les contribuía a mejorar su trabajo y obtener los logros esperados en el proceso educativo. Posteriormente y con base en los criterios vertidos por los docentes se procedió a la adecuación y acoplamiento de la guía del EJS con los contenidos establecidos en el sílabo y en el programa analítico de la asignatura de análisis matemático III, especialmente con los problemas aplicativos de carácter práctico que involucra la materia.

A continuación, se procedió a determinar población y muestra de la investigación. La muestra fue repartida en dos grupos, donde el primero sería el grupo control, en el cual se mantenía la metodología tradicional del proceso educativo. El segundo grupo sería el grupo de intervención, en el cual se aplicaría la nueva metodología del proceso educativo, basada en el EJS. Finalizado el ciclo académico, se procedió a la evaluación de los resultados académicos alcanzados con los dos grupos, y al análisis e interpretación de los mismos para determinar los resultados obtenidos.

Dentro del trabajo desarrollado, la guía adaptada para la aplicación del EJS consistía en un manual de usuario, acompañado de varios problemas físicos, seleccionados como aplicaciones prácticas que tienen que ver con el modelado matemático, cuya solución implicaba el uso de la temática abordada en la asignatura de análisis matemático III, como es el caso de las ecuaciones diferenciales. La guía elaborada contiene información relevante que orienta a la población estudiantil en su preparación académica para un mejor aprendizaje, y por ende, para que logren un buen resultado evaluativo en la materia. 
Para la implementación del EJS, se partió de una revisión previa de la guía elaborada y de la fundamentación matemática requerida para la resolución de los problemas que se plantean. A continuación, se procedió a la revisión de las herramientas que contiene el software y la aplicación con problemas teóricos básicos, lo que permitía posteriormente utilizar esta herramienta para resolver problemas propios de la temática abordada en la asignatura, principalmente la aplicación a problemas que se deban abordar por medio de ecuaciones diferenciales con condiciones de frontera.

\section{Técnica de análisis de datos}

Una vez finalizado el ciclo y obtenido los resultados académicos de cada uno de los integrantes de los dos grupos (control e intervención), se procedió a realizar una codificación abierta, ya que esta ofrece un panorama de los fenómenos relevantes de los datos recabados. La codificación abierta es un proceso analítico por medio del cual se identifican los conceptos, aplicando etiquetas verbales a unidades de sentido, con el fin de obtener las propiedades y dimensiones de los datos (Strauss y Corbin, 2002). De esta forma se accedió a los elementos relevantes para la investigación.

\section{Validación del instrumento}

De manera tradicional, la validez de un instrumento se ha definido como el grado en que calcula lo que está diseñado para medir, indicando si se cumplen adecuadamente los fines para los cuales fue diseñado y construido. Un instrumento puede tener muchas clases de validez, dependiendo de los propósitos específicos para los cuales ha sido diseñado, la población objetivo, las condiciones en que se aplica y el método para determinar la validez (Aiken, 2003). Uno de los tipos de validez más recurrentes es la validez de contenido, o el grado en que el instrumento es representativo o cubre suficientemente el contenido en cuestión. Es decir, este tipo de validez hace referencia a la relevancia y representatividad del contenido del instrumento con respecto a la extensión de todo el dominio que se pretende abarcar (Wenk-Sormaz, 2005).

De este modo, la validez de contenido de la herramienta metodológica aplicada, se la realizó con base en los criterios vertidos por los docentes participantes en la investigación, y a través de los resultados académicos obtenidos en los dos grupos de trabajo. Especial participación del área de matemáticas, en donde se evaluó si el contenido del instrumento cumple con los objetivos para los que fue diseñado, teniendo como indicadores pertinencia, suficiencia y claridad. Pertinencia, entendemos la adecuación o el sentido de algo que encaja perfectamente en un determinado contexto. Suficiencia, se entiende la capacidad o aptitud mínima para realizar algo. Claridad, es la distinción con la que, a través de los sentidos, una persona percibe las sensaciones o las ideas.

Para validar la herramienta aplicada con el EJS, se realizaron los siguientes pasos:

- Selección de docentes: Por sugerencia metodológica, se optó por utilizar la opinión de 4 docentes para este proceso de validación.

- Diseño de pauta de evaluación en el proceso de aplicación.

- Evaluación del instrumento: se contactó a cada uno de los docentes seleccionados cursando la invitación formal a participar del proceso y posteriormente se les hizo entrega de la pauta de evaluación y el instrumento.

- Aplicación de un Índice de Aprobación de contenido tomando como referencia el sílabo y el seguimiento al sílabo.

\section{Población y muestra}

La realización de esta investigación toma como población referencial a los estudiantes que cursan la asignatura de Análisis Matemático III de la Facultad de Mecánica, es decir, a aquellos estudiantes que hayan aprobado los requisitos previstos para poder cursar esta materia y que tengan conocimientos de Informática.

Tabla 1. Población del estudio

\begin{tabular}{lll}
\hline ESTRATOS & POBLACIÓN & PORCENTAJE \\
\hline Escuela de Ing. Mecánica & 65 & $25 \%$ \\
\hline Escuela de Ing. Automotriz & 70 & $27 \%$ \\
\hline Escuela de Ing. Industrial & 60 & $23 \%$ \\
\hline $\begin{array}{l}\text { Escuela de Ing. de } \\
\text { Mantenimiento }\end{array}$ & 65 & $25 \%$ \\
\hline Total & 260 & $100 \%$ \\
\hline
\end{tabular}

Fuente: elaboración propia con información suministrada por Secretaría académica ESPOCH. 
Dadas las características de la población considerada para la investigación, la muestra consistió en los dos paralelos de estudiantes de la Escuela de Ingeniería de Mantenimiento, basado en el muestreo no probabilístico casual o incidental, puesto que se tiene un trabajo conjunto entre los dos profesores que dictan la materia de análisis matemático III, en el período académico Octubre 2016 a Marzo 2017; basando de esta forma la investigación en una aplicación de un diseño experimental por bloques (Walpole et. al, 2012).

Tabla 2. Muestra para el estudio

\begin{tabular}{ccc}
\hline ESTRATOS & POBLACIÓN & PORCENTAJE \\
\hline Paralelo 1 & 30 & $46 \%$ \\
\hline Paralelo 2 & 35 & $54 \%$ \\
\hline Total & 65 & $100 \%$
\end{tabular}

Fuente: elaboración propia con información suministrada por Secretaría académica ESPOCH.

\section{Exposición y discusión de resultados de desempeño estudiantil}

El desempeño estudiantil, se analizó con base en las calificaciones obtenidas por los estudiantes en las actividades realizadas, individualmente y por grupos. Sobre estas, se determina la existencia o no, de diferencias significativas entre las clases que continuaron con métodos tradicionales y las que aplicaron la nueva propuesta, es decir, con herramientas informáticas.

De este modo, el análisis cuantitativo se realiza sobre la comparación de los resultados de las evaluaciones obtenidas por los estudiantes, considerando tanto el proceso enseñanza-aprendizaje tradicional aplicada al grupo control y la metodología planteada en el presente trabajo aplicada al grupo de intervención. Las evaluaciones realizadas durante el proceso educativo fueron realizadas tomando en consideración las destrezas alcanzadas por los estudiantes, con criterio de desempeño al momento de evaluar el aprovechamiento estudiantil en cada una de sus facetas de aprendizaje significativo de la asignatura de análisis matemático III.

Los resultados del análisis realizado se presentan en la siguiente tabla:
Tabla 3. Desempeño estudiantil por categorías y grupos de contraste

\begin{tabular}{llcccc}
\hline \multirow{2}{*}{ Evaluación } & \multicolumn{4}{c}{ Grupos de contraste } \\
\cline { 3 - 6 } & \multicolumn{3}{c}{ Sin EJS } & \multicolumn{2}{c}{ Con EJS } \\
\cline { 2 - 6 } & $\begin{array}{l}\text { No alcanza los } \\
\text { aprendizajes. }\end{array}$ & 10 & $33.3 \%$ & 5 & $14.3 \%$ \\
\cline { 2 - 6 } & $\begin{array}{l}\text { Está próximo a alcanzar } \\
\text { aprendizajes. }\end{array}$ & 8 & $26.7 \%$ & 7 & $20.0 \%$ \\
\cline { 2 - 6 } & $\begin{array}{l}\text { Alcanza los } \\
\text { aprendizajes. }\end{array}$ & 8 & $26.7 \%$ & 13 & $37.1 \%$ \\
\cline { 2 - 6 } & $\begin{array}{l}\text { Domina y supera } \\
\text { aprendizajes. }\end{array}$ & 4 & $13.3 \%$ & 10 & $28.6 \%$ \\
\hline
\end{tabular}

Fuente: Ficha técnica seguimiento.

\section{Interpretación}

$\mathrm{Al}$ medir los resultados entre los dos cursos resalta como el 33.3\% de los estudiantes del grupo de control no alcanzan los niveles de aprendizaje requeridos, en tanto que los estudiantes que aplican el simulador (grupo de intervención), este porcentaje se reduce a un $14.3 \%$. Otro aspecto importante es que los estudiantes que dominan el aprendizaje, con la implementación del EJS son del $28,6 \%$ superando en más del doble a los estudiantes que no utilizaron el simulador, que corresponde al 13,3\%.

De este modo, se aprecia como el rendimiento académico de los estudiantes del grupo de intervención es mayor que el que presenta el grupo control. Surge la pregunta si efectivamente la introducción del EJS, explica esta diferencia. La hipótesis es afirmativa, ya que el EJS es una herramienta que ayuda al docente a desarrollar ciertas destrezas y habilidades en los estudiantes (como la visualización del comportamiento de los sistemas físicos mediante la simulación de los problemas en estudio), que no se logra con la metodología tradicional, permitiendo un aprendizaje más significativo.

\section{Comprobación de la hipótesis}

Hipótesis:

$\mathrm{H}_{1}$ : La Implementación de simulaciones con EJS, incide en el rendimiento académico de los estudiantes de análisis matemático III.

$\mathrm{H}_{0}:$ La Implementación de simulaciones con EJS, no incide en el rendimiento académico de los estudiantes de Análisis Matemático III. 
Para comprobar las hipótesis se utiliza el estadístico T Student, para dos muestras independientes. El mismo se representa a partir de:

$t=\frac{M_{1}-M_{2}}{\sqrt{s_{1}^{2} / n_{1}+{ }^{s_{2}^{2}} / n_{2}}}$

Donde $M_{1}$ y $M_{2}$ son las medias de ambos grupos, $s$ la desviación típica y $n$ el número de casos. Esto nos permitirá determinar si en promedio el desarrollo de destrezas es significativamente diferente. Este contraste se realiza a un nivel de confianza del $95 \%$. Este tipo de pruebas se suelen usar cuando se elige una muestra de individuos que han seguido cierto tratamiento (por ejemplo, un grupo control, paralelo A) y otra muestra que recibe otro tratamiento (grupo de intervención, en el que se la aplica guía de simulaciones con EJS).

Tabla 4. Descriptivos notas grupo de investigación

\begin{tabular}{cccccc}
\hline & & \multicolumn{4}{c}{ ESTADístICOS DE GRUPO } \\
\cline { 3 - 6 } & $\mathbf{N}$ & Media & $\begin{array}{c}\text { Desviación } \\
\text { tip. }\end{array}$ & $\begin{array}{c}\text { Error } \\
\text { tip. de la } \\
\text { media }\end{array}$ \\
\hline \multirow{2}{*}{ Grupos } & $\begin{array}{c}\text { Sin } \\
\text { Implementación } \\
\text { A }\end{array}$ & 30 & 4.6667 & 1.799 & 0.465 \\
\cline { 2 - 5 } & $\begin{array}{c}\text { Con } \\
\text { Implementación } \\
\text { B }\end{array}$ & 35 & 7.2286 & 2.016 & 0.341 \\
\hline
\end{tabular}

Fuente: elaboración propia sobre acta notas de evaluación.

Tabla 5. Distribución, prueba de hipótesis

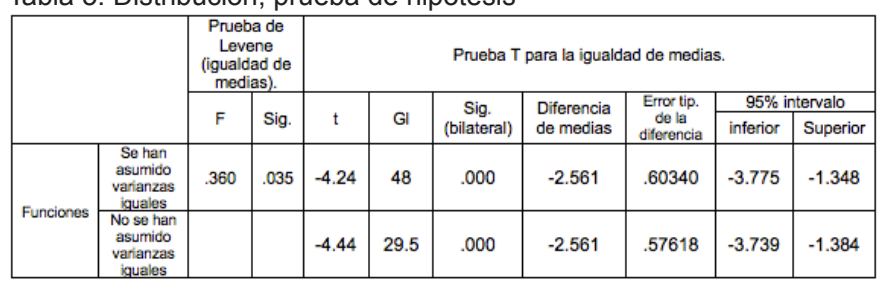

Fuente: elaboración propia sobre acta notas de evaluación

\section{Análisis de los grupos}

La prueba de Levene permite establecer si las varianzas de las medias son iguales o no entre los grupos de contraste, considerando como criterio de evaluación el valor de la significancia de dicha prueba, la cual toma como valor de análisis el 0.05. De los resultados de la prueba de hipótesis realizada se tiene que el valor de la significancia de la prueba de Levene es de 0.035 , por lo que las varianzas no son iguales, razón por la que elegimos el resultado de la prueba T, para contrastar la hipótesis bajo el criterio "no se han asumido varianzas iguales" (Walpole et al., 2012).

De la prueba T realizada, la significación bilateral es de 0.000 (menor que 0.05), por lo que podemos afirmar que si existen diferencias significativas entre ambos grupos, es decir, la implementación del software EJS en el proceso de enseñanza-aprendizaje de la asignatura análisis matemático III, si influye significativamente en el rendimiento académico de los estudiantes objeto de la investigación, por lo que se aprueba $\mathrm{H}_{1}$ y se rechaza $\mathrm{H}_{0}$, esto se determina observando el intervalo de confianza para la diferencia de las dos metodologías aplicadas. Para realizar el análisis estadístico se utilizó el software SPSS.

Según los resultados del rendimiento académico obtenidos en la investigación y el tratamiento estadístico realizado con dicha información, se logra dar respuesta a la hipótesis de investigación: ¿la implementación del EJS incide en el rendimiento académico de los estudiantes de análisis matemático III? La respuesta positiva se explica sin duda por el ambiente interactivo y colaborativo que el software promociona. Los alumnos del grupo de intervención mejoran su rendimiento académico y superan con un promedio de 7.23 en los resultados globales del curso, al promedio de 4.67 que obtuvo el grupo control" (promedio que afecta el rendimiento académico que al final perjudica la aprobación de la asignatura).

A esto se suma que al evaluar la ficha de comportamiento estudiantil, en promedio más del $50 \%$ de los estudiantes del grupo de intervención elevan sus capacidades y destrezas en la asignatura de análisis matemático III, quienes desarrollan un razonamiento apuntalado en la lógica y en la creatividad, lo que les permite plantear nuevos problemas relacionados con su campo de estudio.

La evaluación del proceso educativo durante el tiempo que se desarrolló el trabajo investigativo, se realizó para los dos grupos a través de pruebas objetivas aplicadas independientemente a cada grupo y talleres, además en el grupo de intervención se realizó simulaciones de problemas diferentes, lecciones individuales bajo el análisis del programa EJS, para luego comparar los resultados obtenidos con los del grupo control en base de las notas finales. De acuerdo a los resultados académicos obtenidos al final del ciclo en el que se desarrolló este trabajo, se pudo determinar que el criterio de "pertinencia" 
es aplicable al estudiante mejor evaluado, quien tuvo un rendimiento del 93\%. Sin duda, la implementación de software EJS resulta pertinente a la realidad de la temática.

\section{Conclusiones}

La aproximación teórica - práctica de esta investigación, permitió identificar aquellos elementos que son de utilidad, tanto para el docente como para el estudiante, en el proceso de enseñanza aprendizaje de los contenidos de una materia como "análisis matemático III" y que pueden ser generalizados a otras temáticas de la matemática a través del EJS.

Del rendimiento académico alcanzado por los dos grupos de estudiantes participantes de la investigación, se concluye la existencia de diferencias significativas entre el grupo control y el grupo de intervención, es decir, la implementación de la guía y el software EJS en el proceso de enseñanza-aprendizaje de análisis matemático III, sí influye positivamente en el rendimiento académico.

La socialización de esta herramienta informática, permitiría replicar la metodología aplicada y mejorar la comprensión de las temáticas y problemas resueltos con la implementación del software EJS. Con la experiencia adquirida, se ha generado un texto guía que permite aplicar esta metodología en cualquier carrera de cualquier universidad donde conste en su malla curricular la asignatura de análisis matemático III, o sus equivalentes de acuerdo con sus contenidos temáticos. Debido al tipo de muestreo aplicado en este trabajo y su implicación, sería conveniente que el proceso de investigación se continúe y se aplique a una población constituida por todas varias carreras, que poseen en sus mallas curriculares la asignatura de análisis matemático III, o sus equivalentes.

\section{Referencias}

1. Aiken, L. (2003). Tests psicológicos y evaluación. $11^{\mathrm{a}}$ Edición. Editorial Pearson Educación. México.

2. Strauss, A. y Corbin, J. (2002). Bases de la Investigación Cualitativa. Técnicas y procedimientos para desarrollar la teoría fundamentada. Editorial Contus, Facultad de Enfermería de la Universidad de Antioquia. Medellín (Colombia). Recuperado de: $\quad$ https://diversidadlocal.files.wordpress. com/2012/09/bases-investigacion-cualitativa.pdf

3. Walpole, R., Myers R., Myers S., y Ye, K. (2012). Probabilidad y estadística para ingeniería y ciencias. 9a Edición. Editorial Pearson Educación, México.

4. Wenk-Sormaz, H. (2005). Meditation can reduce habitual responding. Alternative therapies in health and medicine, 11(2), pp. 42-59. 